\title{
E2E NLG Challenge Submission: Towards Controllable Generation of Diverse Natural Language
}

\author{
Henry Elder \\ Adapt Centre / DCU \\ henry.elder@adaptcentre.ie
}

Alexander O'Connor

Pivotus Ventures Inc.

alexanderepivotusventures. com

\author{
Sebastian Gehrmann \\ Harvard NLP \\ gehrmanneg.harvard.edu \\ Qun Liu \\ Adapt Centre / DCU \\ qun. liuladaptcentre. ie
}

\begin{abstract}
In natural language generation (NLG), the task is to generate utterances from a more abstract input, such as structured data. An added challenge is to generate utterances that contain an accurate representation of the input, while reflecting the fluency and variety of human-generated text. In this paper, we report experiments with NLG models that can be used in task oriented dialogue systems. We explore the use of additional input to the model to encourage diversity and control of outputs. While our submission does not rank highly using automated metrics, qualitative investigation of generated utterances suggests the use of additional information in neural network NLG systems to be a promising research direction.
\end{abstract}

\section{Introduction}

Natural Language Generation (NLG) is a broad field, ranging from text-to-text translation to experiments in computational poetry (Gatt and Krahmer, 2018). Whether the task is to summarize, translate, or entertain, a core challenge is doing so in a manner that is compatible with human needs and preferences.

Formally, NLG systems aim to create utterances from a set of abstract inputs. These inputs can be closely aligned, e.g. machine translation (Sutskever et al., 2014), or require significant abstractive reasoning, as in summarization or datato-text tasks (See and Manning, 2017; Wiseman et al., 2017). Traditionally NLG systems have followed a rule-based approach (Reiter and Dale, 2000). While robust, these systems are noted to generate repetitive and stilted output, which can

Meaning Representation
name[The Wrestlers]
eatType[restaurant]
food[Japanese]
priceRange[more than $£ 30]$
area[riverside]
familyFriendly[no]
near[Raja Indian Cuisine]
additionalWords[looking adults offerings
really try good prices situated]
Generated utterance
If you're looking for an adults only
Japanese restaurant, try The Wrestlers. It
is really good and situated near Raja In-
dian Cuisine. The prices are more than
$£ 30$.

Table 1: Utterance generated with a novel dialogue act containing additional words

make interacting with rule based systems a tedious experience (Wen et al., 2015).

Data driven models using deep neural networks have achieved state-of-the-art results in many NLG tasks/datasets such as RoboCup, Weathergov, SF Hotels/Restaurants and AMR-to-text (Mei et al., 2016; Wen et al., 2016; Konstas et al., 2017). However Sharma et al. (2017) notes that high performance on datasets such as Wen et al. (2015)'s SF Restaurant indicates they no longer pose a sufficient challenge and that the community ought to progress to using larger and more complex datasets.

Two new crowd sourced datasets, each containing tens of thousands of examples and focusing on complex sentence structures, have been recently released; WebNLG and E2E (Colin et al., 2016; Novikova et al., 2017). This paper focuses on the E2E dataset which was created using a new 
methodology to maximize both the quality of collected utterances as well as their naturalness and variety (Novikova et al., 2016).

Wei et al. (2017) note that neural networks learning from highly unaligned datasets have trouble choosing between equally plausible outputs and tend towards short and less meaningful outputs. They suggest that the number of plausible outputs can be decreased by providing additional information to the model. In Table 1 we augment the meaning representation (MR) with a novel dialogue act (DA) containing additional words to be included in the generated utterance. By conditioning the output on these words the model has managed to generate an utterance with a complex sentence structure and wide vocabulary.

Our contribution is to propose a pipeline system. Additional words are sampled from a secondary model which uses DAs from a given MR as inputs. These additional words are put into a new DA and added to the existing MR, as shown in Table 1. The augmented MR is then used as input to a model which generates the final utterance.

The approach of augmenting the source sequence takes inspiration from recent work in paraphrase generation (Guu et al., 2017) and generating structured queries from natural language (Zhong et al., 2017). As noted by Sharma et al. (2016) delexicalization can often lead to grammatically incorrect sentences. We opt instead to use a pointer network (Vinyals et al., 2015) which allows the model to copy tokens directly from the source sequence into the generated utterance. The model does not perform well relative to the baseline and this is possibly due to the failure of the secondary model to generate appropriate additional words. Improving upon the pipeline system remains an area of active research for us.

\section{System Description}

Here we present details of the pipeline system. First we describe how the training data for the pointer network with additional words model is constructed. This is followed by an explanation of the additional word generator which uses DAs from a given MR as input.

Typical approaches to generating diverse outputs focus on objective functions that affect the decoding step (Li et al., 2015). Our approach of augmenting the input sequence is similar to previous work on common sense dialogue models (Young et al., 2017) and content-introducing text generation (Mou et al., 2016). Other approaches to controllable text generation have focused on more abstract inputs. Language models which generate text about a specific topic, product, person, sentiment (Li et al., 2016; Tang et al., 2016; Fan et al., 2017; Dong et al., 2017).

\subsection{Additional words model}

We augment the MR with an extra DA containing additional words to be included in the generated sentence. To obtain the data for this we looked at each target sentence and, using a set of rules, determined what words the model would learn to include. These selected words were added to the source sequence inside a custom DA. This ability of the model to accept additional words ensured that we would have both diversity of outputs and fine grained control over those outputs at test time.

For our additional words model we extracted tokens from the target sequence that adhered to the following set of rules:

- Not part of a list of stopwords

- Does not appear in the source sequence or meaning representation

- Does not contain punctuation or numbers

After the original list was compiled we removed the most frequently appearing token located and any tokens which occurred less than 6 times.

Table 2 contains an example of an augmented MR and utterance pair used for training.

\begin{tabular}{l} 
Source sequence \\
\hline name[The Vaults] \\
eatType[pub] \\
priceRange[more than $£ 30]$ \\
customer rating[5 out of 5] \\
near[Café Adriatic] \\
additionalWords[star Prices start] \\
Target sequence \\
The Vaults pub near Café Adriatic has a 5 \\
star rating. Prices start at $£ 30$.
\end{tabular}

Table 2: Example from the additional words model training set 


\subsubsection{Generating additional words}

The unique contents of each DA in the MR are treated as a single token. We omit the name and near DAs as they were observed to have little correlation with the semantics of the additional words chosen. The model attempts to correlate specific DAs with the additional words that appear in target sentences. An example of the source and target sequences used for training are shown in Table 3. We use a sequence-to-sequence network with attention as the model.

Additional words are sampled from the model. We scale the final output layer of the model before applying softmax and sampling tokens for the generated utterance. The value used for scaling is known as temperature. Higher values of temperature lead to more diverse outputs. Temperature values close to 0 lead to the model choosing more conservative outputs. We use values of 0.9 to 1.1 , to encourage the generation of a more diverse set of additional words.

\begin{tabular}{l} 
Source sequence \\
\hline pub \\
more_than_f30 \\
5_out_of_5 \\
Target sequence \\
\hline star Prices start
\end{tabular}

Table 3: Example pair used for training the additional word generator

\section{Experiments}

The data set was tokenized using the NLTK port of the moses tokenizer with aggressive hyphen splitting. For each DA a custom start and stop token was added to the source sequence; e.g. __name_start_- The Vaults __name_end _-_

The models used were from the OpenNMT-py library (Klein et al., 2017). Our model architecture contains 2 layers of bidirectional recurrent neural networks (RNN) with long short-term memory (LSTM) cells (Hochreiter and Schmidhuber, 1997). We use 500 hidden units for the encoder and decoder layer, and 500 units for the word vectors which are learned jointly across the whole model. We add dropout of 0.3 applied between the LSTM stacks.

The models are trained using Adam (Kingma and $\mathrm{Ba}, 2014$ ) with learning rate 0.001 and learn- ing rate decay of 0.5 applied after 8 epochs. The models were trained for 10 epochs and the best performing checkpoint on the development set was chosen.

The exploration and choice of hyperparameters was aided by the use of Bayesian hyperparameter optimization platform SigOpt (2014).

\section{Results \& Discussion}

We report results using automated evaluation metrics; BLEU (Papineni et al., 2002), NIST (Przybocki et al., 2009), METEOR (Lavie and Agarwal, 2007), and ROUGE-L (Lin, 2004). Table 4 shows the performance of the baseline relative to our models using both sample additional words and those extracted from target sentences, these are the gold standard additional words. The baseline model is TGen, a sequence-to-sequence model with attention (Dušek and Jurčíček, 2016).

The model using extracted additional words performs better in almost all metrics. The poor performance of models using sampled words versus gold standard words highlights an issue with the generation of additional words. These results maintain their relative ranking in the test set as shown in Table 5.

Human evaluation was carried out on the primary systems. The two metrics used were Quality; which measures grammatical correctness and overall adequacy in the context of the MR, and Naturalness; could the utterance have been produced by a native speaker. Crowd workers were used to collect pairwise comparisons for each system. Systems were ranked using the TrueSkill algorithm (Sakaguchi et al., 2014). Our model ranked 4th, below the baseline which came in 2nd, as shown in Table 4 (Dušek et al., 2018)

Automated evaluation and subsequent human evaluation results show our additional words model performs poorly relative to the baseline. A manual observation of the model's outputs reveal many errors such as repeated phrases and occasionally absent or incorrect information. We include a collection of generated utterances from the test set in table 7 to highlight areas where the model performs both well and poorly relative to the baseline.

Utterances from the baseline model tend to be more consistent but when viewed over many hundreds of samples this can be dry and repetitive. In most cases the baseline model appears to have 


\begin{tabular}{llllll} 
Model & BLEU & NIST & METEOR & ROUGE-L & CIDEr \\
\hline Additional words - temperature 1.1 & 0.5307 & 7.1738 & 0.4108 & 0.6112 & 1.5658 \\
Additional words - temperature 1.0 & 0.5574 & 7.4078 & 0.4171 & 0.6308 & 1.6380 \\
Additional words - temperature 0.9 & 0.5659 & 7.5196 & 0.4209 & 0.6327 & 1.7652 \\
Baseline & 0.6925 & 8.4781 & 0.4703 & 0.7257 & $\mathbf{2 . 3 9 8 7}$ \\
Additional words - extracted from target & $\mathbf{0 . 7 3 8 1}$ & $\mathbf{9 . 9 4 3 5}$ & $\mathbf{0 . 4 7 2 6}$ & $\mathbf{0 . 7 5 0 8}$ & 2.2858
\end{tabular}

Table 4: Dev set results

\begin{tabular}{llllll} 
Model & BLEU & NIST & METEOR & ROUGE-L & CIDEr \\
\hline Additional words - temperature 1.1 & 0.5092 & 7.1954 & 0.4025 & 0.5872 & 1.5039 \\
Additional words - temperature 1.0 & 0.5265 & 7.3991 & 0.4095 & 0.5992 & 1.6488 \\
Additional words - temperature 0.9 & 0.5573 & 7.7013 & 0.4154 & 0.6130 & 1.8110 \\
Baseline & $\mathbf{0 . 6 5 9 3}$ & $\mathbf{8 . 6 0 9 4}$ & $\mathbf{0 . 4 4 8 3}$ & $\mathbf{0 . 6 8 5 0}$ & $\mathbf{2 . 2 3 3 8}$
\end{tabular}

Table 5: Test set results

\begin{tabular}{|c|c|c|}
\hline Model & Naturalness & Quality \\
\hline Baseline & 2nd & 2nd \\
\hline $\begin{array}{l}\text { Additional words } \\
\text { temperature } 1.1\end{array}$ & 4th & 4th \\
\hline
\end{tabular}

Table 6: True skill clusters

learned its own simple templates for generating utterances from an MR. The following is an example of the template-like output the baseline produces if provided with all 8 possible DAs; "[name] is a [food] [eatType] near [near] in the [area]. It has a [customer rating] and a price range of [price range]. It is [family friendly]." While the baseline model outperformed rule based systems in the E2E challenge, its generated utterances do not appear to fully reflect the diversity of the dataset which has been collected.

\section{Future Work}

Many verbalization issues in the additional word model arise due to a conflict between an additional word and the existing DAs in the MR. This can be seen in some of the examples in Table 7. The model used for generating additional words could be improved substantially. Increasing the minimum frequency of occurrence for additional words in the training data may give the model more examples from which to better learn correct syntax. The pointer network with additional words model also suffers from an issue, common with pointer networks, in which source tokens are incorrectly repeated in the generated utterance. One way to handle this would be to have a second stage of training with a coverage loss as in See and Man- ning (2017).

\section{Conclusion}

We proposed the use of an additional DA to improve the diversity and level of control over utterances. Results show both the underlying network and the method used for generating additional words could be improved. Observation of generated samples show this approach has the potential to yield high quality and varied responses.

\section{References}

Emilie Colin, Claire Gardent, M Yassine, and Shashi Narayan. 2016. The WebNLG Challenge : Generating Text from DBPedia Data. The 9th International Conference on Natural Language Generation.

Li Dong, Shaohan Huang, Furu Wei, Mirella Lapata, Ming Zhou, and Ke Xu. 2017. Learning to Generate Product Reviews from Attributes.

Ondrej Dušek, Jekaterina Novikova, and Verena Rieser. 2018. Findings of the E2E NLG challenge.

Ondej Dušek and Filip Jurčíček. 2016. Sequence-toSequence Generation for Spoken Dialogue via Deep Syntax Trees and Strings.

Angela Fan, David Grangier, and Michael Auli. 2017. Controllable Abstractive Summarization.

Albert Gatt and Emiel Krahmer. 2018. Survey of the state of the art in natural language generation: Core tasks, applications and evaluation. Journal of Artificial Intelligence Research, 61(c):1-64.

Kelvin Guu, Tatsunori B Hashimoto, Yonatan Oren, and Percy Liang. 2017. Generating Sentences by Editing Prototypes. 2. 


\begin{tabular}{|c|c|}
\hline Additional words DA & $\begin{array}{l}\text { name[The Cricketers] eatType[coffee shop] customerrating[low] fami- } \\
\text { lyFriendly[no] near[Express by Holiday Inn] } \\
\text { additionalWords[satisfaction considered Join] }\end{array}$ \\
\hline Additional words model & $\begin{array}{l}\text { The Cricketers is a coffee shop near Express by Holiday Inn. It has a low } \\
\text { customer satisfaction rating and is not considered family-friendly. Join Us. } \\
\text { The Cricketers is a coffee shop near Express by Holiday Inn. It has a low } \\
\text { customer rating and is not family-friendly. }\end{array}$ \\
\hline $\begin{array}{l}\text { MR } \\
\text { Additional words DA }\end{array}$ & $\begin{array}{l}\text { name[Zizzi] eatType[coffee shop] area[riverside] } \\
\text { additionalWords[Located along river side offering snacks] }\end{array}$ \\
\hline $\begin{array}{l}\text { Additional words model } \\
\text { Baseline }\end{array}$ & $\begin{array}{l}\text { Located along the river side, Zizzi is a coffee shop offering snacks . } \\
\text { Zizzi is a coffee shop in the riverside area with a price range of less than } 20 \text {. }\end{array}$ \\
\hline $\begin{array}{l}\text { MR } \\
\text { Additional words DA }\end{array}$ & $\begin{array}{l}\text { name[The Mill] eatType[restaurant] food[English] priceRange[high] cus- } \\
\text { tomerrating[average] area[riverside] familyFriendly[yes] near[Café Rouge] } \\
\text { additionalWords[offers fare higher bracket] }\end{array}$ \\
\hline $\begin{array}{l}\text { Additional words model } \\
\text { Baseline }\end{array}$ & $\begin{array}{l}\text { The Mill is a family friendly restaurant that offers English fare in the higher } \\
\text { price bracket. It is located near Café Rouge in the riverside area . } \\
\text { The Mill is an english restaurant near Café Rouge in the riverside area. It is } \\
\text { children friendly and has a high price range and an average customer rating. }\end{array}$ \\
\hline MR & $\begin{array}{l}\text { name[Strada] eatType[coffee shop] customerrating[1 out of 5] near[All Bar } \\
\text { One] } \\
\text { additionalWords[try perfect however] }\end{array}$ \\
\hline $\begin{array}{l}\text { Additional words model } \\
\text { Baseline }\end{array}$ & $\begin{array}{l}\text { For a coffee shop near All Bar One, try Strada. It has a perfect customer rating } \\
\text { of } 1 \text { out of } 5 \text { however. } \\
\text { Strada is a coffee shop near All Bar One with a customer rating of } 1 \text { out of } 5 \text {. }\end{array}$ \\
\hline MR & $\begin{array}{l}\text { name[Blue Spice] eatType[coffee shop] customerrating[average] near[Burger } \\
\text { King] } \\
\text { additionalWords[neat] }\end{array}$ \\
\hline $\begin{array}{l}\text { Additional words model } \\
\text { Baseline }\end{array}$ & $\begin{array}{l}\text { Blue Spice is a coffee shop located neat Burger King. } \\
\text { Blue Spice is a coffee shop near Burger King. It has an average customer } \\
\text { rating. }\end{array}$ \\
\hline $\begin{array}{l}\text { MR } \\
\text { Addi }\end{array}$ & $\begin{array}{l}\text { name[Wildwood] eatType[pub] area[riverside] } \\
\text { additionalWords[Located river serving] }\end{array}$ \\
\hline $\begin{array}{l}\text { Additional words model } \\
\text { Baseline }\end{array}$ & $\begin{array}{l}\text { Located near the river, Wildwood is a pub ser } \\
\text { Wildwood is a pub by the riverside. }\end{array}$ \\
\hline
\end{tabular}

Table 7: Example generated utterances using MRs from the test set

Sepp Hochreiter and J Schmidhuber. 1997. Long Short-Term Memory. Neural Computation, 9(8):1735-1780.

Diederik P. Kingma and Jimmy Ba. 2014. Adam: A Method for Stochastic Optimization.

Guillaume Klein, Yoon Kim, Yuntian Deng, Josep Crego, Jean Senellart, and Alexander M. Rush. 2017. OpenNMT: Open-source Toolkit for Neural Machine Translation. pages 67-72.

Ioannis Konstas, Srinivasan Iyer, Mark Yatskar, Yejin Choi, and Luke Zettlemoyer. 2017. Neural AMR: Sequence-to-Sequence Models for Parsing and Generation. pages 146-157.

Alon Lavie and Abhaya Agarwal. 2007. Meteor: An
Automatic Metric for MT Evaluation with High Levels of Correlation with Human Judgments. In Proceedings of the Second Workshop on Statistical Machine Translation, StatMT '07, pages 228-231, Stroudsburg, PA, USA. Association for Computational Linguistics.

Jiwei Li, Michel Galley, Chris Brockett, Jianfeng Gao, and Bill Dolan. 2015. A Diversity-Promoting Objective Function for Neural Conversation Models.

Jiwei Li, Michel Galley, Chris Brockett, Georgios P Spithourakis, Jianfeng Gao, and Bill Dolan. 2016. A Persona-Based Neural Conversation Model. pages 994-1003.

C Y Lin. 2004. Rouge: A package for automatic evaluation of summaries. Proceedings of the workshop 
on text summarization branches out (WAS 2004), (1):25-26.

Hongyuan Mei, Mohit Bansal, and Matthew R. Walter. 2016. What to talk about and how? Selective Generation using LSTMs with Coarse-to-Fine Alignment. Proceedings of the 15th Annual Conference of the North American Chapter of the Association for Computational Linguistics: Human Language Technologies (NAACL-HLT'16(, pages 1-11.

Lili Mou, Yiping Song, Rui Yan, Ge Li, Lu Zhang, and Zhi Jin. 2016. Sequence to Backward and Forward Sequences: A Content-Introducing Approach to Generative Short-Text Conversation.

Jekaterina Novikova, Ondrej Dušek, and Verena Rieser. 2017. The E2E dataset: New challenges for end-toend generation. ArXiv:1706.09254.

Jekaterina Novikova, Oliver Lemon, and Verena Rieser. 2016. Crowd-sourcing NLG Data: Pictures Elicit Better Data. (1).

Kishore Papineni, Salim Roukos, Todd Ward, and WeiJing Zhu. 2002. BLEU: A Method for Automatic Evaluation of Machine Translation. In Proceedings of the 40th Annual Meeting on Association for Computational Linguistics, ACL '02, pages 311-318, Stroudsburg, PA, USA. Association for Computational Linguistics.

Franois Portet, Ehud Reiter, Albert Gatt, Jim Hunter, Somayajulu Sripada, Yvonne Freer, and Cindy Sykes. 2009. Automatic generation of textual summaries from neonatal intensive care data. Artificial Intelligence, 173(7-8):789-816.

Mark Przybocki, Kay Peterson, Sbastien Bronsart, and Gregory Sanders. 2009. The NIST 2008 Metrics for machine translation challenge-overview, methodology, metrics, and results. Machine Translation, 23(2):71-103.

Ehud Reiter and Robert Dale. 2000. Building Natural Language Generation Systems. Cambridge University Press, New York, NY, USA.

Keisuke Sakaguchi, Matt Post, and Benjamin Van Durme. 2014. Efficient Elicitation of Annotations for Human Evaluation of Machine Translation. Proceedings of the Ninth Workshop on Statistical Machine Translation (WMT'14), pages 1-11.

Abigail See and Christopher D. Manning. 2017. Get To The Point : Summarization with Pointer-Generator Networks. Association for Computational Linguistics, pages 1-18.

Shikhar Sharma, Layla El Asri, Hannes Schulz, and Jeremie Zumer. 2017. Relevance of Unsupervised Metrics in Task-Oriented Dialogue for Evaluating Natural Language Generation.
Shikhar Sharma, Microsoft Maluuba, Jing He, Adeptmind Kaheer Suleman, Hannes Schulz, and Philip Bachman. 2016. Natural Language Generation in Dialogue using Lexicalized and Delexicalized Data.

Inc. SigOpt. 2014. Sigopt reference manual.

Ilya Sutskever, Oriol Vinyals, and Quoc V. Le. 2014. Sequence to Sequence Learning with Neural Networks. pages $1-9$.

Jian Tang, Yifan Yang, Sam Carton, Ming Zhang, and Qiaozhu Mei. 2016. Context-aware Natural Language Generation with Recurrent Neural Networks.

Oriol Vinyals, Meire Fortunato, and Navdeep Jaitly. 2015. Pointer Networks.

Bolin Wei, Shuai Lu, Lili Mou, Hao Zhou, Pascal Poupart, Ge Li, and Zhi Jin. 2017. Why Do Neural Dialog Systems Generate Short and Meaningless Replies? A Comparison between Dialog and Translation.

Tsung-Hsien Wen, Milica Gaši, Nikola Mrkši, Pei-Hao Su, David Vandyke, and Steve Young. 2015. Semantically Conditioned LSTM-based Natural Language Generation for Spoken Dialogue Systems. pages 1711-1721.

Tsung-Hsien Wen, David Vandyke, Nikola Mrksic, Milica Gasic, Lina M. Rojas-Barahona, Pei-Hao Su, Stefan Ultes, and Steve Young. 2016. A Networkbased End-to-End Trainable Task-oriented Dialogue System. 1:438-449.

Sam Wiseman, Stuart M Shieber, and Alexander M Rush. 2017. Challenges in Data-to-Document Generation.

Chen Xing, Wei Wu, Yu Wu, Jie Liu, Yalou Huang, Ming Zhou, and Wei-Ying Ma. 2016. Topic Augmented Neural Response Generation with a Joint Attention Mechanism. pages 1-9.

Tom Young, Erik Cambria, Iti Chaturvedi, Minlie Huang, Hao Zhou, and Subham Biswas. 2017. Augmenting End-to-End Dialog Systems with Commonsense Knowledge.

Victor Zhong, Caiming Xiong, and Richard Socher. 2017. Seq2SQL: Generating Structured Queries from Natural Language using Reinforcement Learning. 